\title{
EGFR NP_005219.2:p.L861F
}

National Cancer Institute

\section{Source}

National Cancer Institute. EGFR NP 005219.2:p.L861F. NCI Thesaurus. Code C98495.

A change in the amino acid residue at position 861 in the epidermal growth factor receptor protein where leucine has been replaced by phenylalanine. 